\title{
Buffer Management and Hybrid Probability Choice Routing for Packet Delivery in Opportunistic Networks
}

\author{
Daru Pan, Wei Cao, Han Zhang, and Mu Lin \\ School of Physics and Telecommunication Engineering, South China Normal University, \\ Guangzhou 510006, China \\ Correspondence should be addressed to Daru Pan, pandaru@126.com
}

Received 8 April 2012; Accepted 20 May 2012

Academic Editor: Carlo Cattani

Copyright (C) 2012 Daru Pan et al. This is an open access article distributed under the Creative Commons Attribution License, which permits unrestricted use, distribution, and reproduction in any medium, provided the original work is properly cited.

\begin{abstract}
Due to the features of long connection delays, frequent network partitions, and topology unsteadiness, the design of opportunistic networks faces the challenge of how to effectively deliver data based only on occasional encountering of nodes, where the conventional routing schemes do not work properly. This paper proposes a hybrid probability choice routing protocol with buffer management for opportunistic networks. A delivery probability function is set up based on continuous encounter duration time, which is used for selecting a better node to relay packets. By combining the buffer management utility and the delivery probability, a total utility is used to decide whether the packet should be kept in the buffer or be directly transmitted to the encountering node. Simulation results show that the proposed routing outperforms the existing one in terms of the delivery rate and the average delay.
\end{abstract}

\section{Introduction}

Opportunistic networks [1-4] are one of the most emerging communication paradigms in wireless mobile communications where most of the time the path from a source to a destination is unstable and may break and be discovered from time to time $[5,6]$. In this case, how to effectively deliver data based only on occasional encountering of nodes becomes a challenge, since the conventional cannot be adopted straightforwardly. To deal with the unpredictability in connections and network partitions, many routing protocols adopt flooding-based and store-carry-forward routing schemes, such as Epidemic Routing (ER) [7], Spray and Waiting [8-10], PRoPHET [11], and MaxPROP [12], to improve the message delivery, where a node receives packets, stores them in their buffers, carries them while moving, and forwards them to other nodes when they encounter each other. 
Epidemic is one of the first routing schemes, adopting the store-carry-forward paradigm. In Epidemic Routing, packets are disseminated in broadcast mode as infectious diseases spread. This packet-spread will continue until all the nodes have a copy of the packet or its TTL (time-to-live) expires. Although, Epidemic Routing achieves eventual delivery of $100 \%$ of messages, but it makes no attempt to eliminate replication, and the high delivery rate is at the expense of the network resource consumption, such as storage of buffer space and transmission bandwidth $[13,14]$. Performance of ER will get worse when the network traffics congest. Spray and Wait $[8,9,15]$ combines the speed of ER with the simplicity and thriftiness and reliability of direct transmission and makes an effort to perform fewer transmissions by controlling the number of packet copies in spray phase and utilizing direct transmission in wait phase. While in scenarios of a high mobility model (like community-based mobility), the direct transmission based wait phase in Spray and Wait routing has low efficiency in delivery delay and probability. ProPHET [11] presents an estimation-based forwarding scheme to direct the messages to the destination node. The basic operation of ProPHET is similar to that of Epidemic. When two nodes meet each other, each node exchanges its summary vectors and delivery predictability to each other. The delivery predictability in the summary vector is used to make a forwarding decision for the packets' delivery. ProPHET is a single-copy forwarding-based scheme, and the limited copy may result in the performance limitation of the initial probability distribution.

Most of the studies on opportunistic networks have been investigated in the design of efficient routing, but few literature focused on buffer management, which is important for the store-carry-forward paradigm, for example, Epidemic Routing has minimum delivery delay under no buffer constrains, but performs worse than other routings when buffer sizes are limited. Most of the routings use the simple drop-tail policy without taking the buffer management into account. However, how to utilize spatial, temporal, and buffer information to make an optimal decision for delivering the packets is an open issue.

In this paper, we propose a hybrid probability choice routing protocol with buffer management. The main work of the proposed method is (1) to set up a delivery probabilities function based on the continuous encounter duration time and buffer information, (2) to let nodes decide how many copies will be transmitted to the encountering nodes according to their delivery probabilities to the destination in the spray phase, and (3) to combine the buffer utility and delivery probability to construct a total utility and to directly deliver the last copy to the encountering node according to the total utility in the wait phase.

The rest of the paper is organized as follows. We give an overview and detailed information of our algorithm in Section 2. We evaluate our scheme through simulation in Section 3 and draw a conclusion in Section 4.

\section{Design of Probability Choice Routing Protocol with Buffer Management}

\subsection{Network Model}

This paper considers the probabilistically contacted opportunistic networks where the networks consist of nodes representing portable wireless devices held by moving elements such as people or vehicles in a community. We model an opportunistic network as a dynamic set of mobile nodes. Nodes may join and leave the network at any time. In our opportunistic scenario, there are three groups of moving elements: pedestrian, bicycles, and vehicles. Each 
group of moving elements follows the map-based movement model with different speed. We use 30 vehicles following predefined routes, 60 nodes are pedestrians, and 30 nodes are bicycles. The vehicles and bicycles choose random destinations in their reach on the map. The number of different moving elements can be changed, which does not affect the characteristics of basic communication.

Communications are based on pairwise contacts [10,16, 17]. Through the pairwise encountering of mobile elements, data stored in devices are opportunistically forwarded over the network. Nodes are assumed to have homogeneous capability in terms of computation, communication, and storage. Opportunistic forwarding decisions are made without the help of the localization services. Their communication capacity is limited by specific wireless techniques. For example, through Bluetooth, node can contact with each other when each of two nodes enters the other's communication range. Two nodes in the network are neighbors and can transfer data packets bidirectional if they are within the communication range. Data is forwarded in a store-and-forward manner, which allows nodes to store data temporarily until running into a more competent node to further forward them. We consider a multicopy scenario. The network model can be presented concretely as follows. The topology of the networks is modeled as a graph $G=(V, E), V$ is the set of nodes, and $E$ is the set of hyperarcs. Each node in the network can be a source or destination of traffic.

\subsection{Motivation of the Protocol}

The core of the processing is how to rank the relay nodes based on the measurement of the delivery predictability and buffer utility. Addressing the above issue, each node records its location and context to a historical information database. Nodes renew their routing passively and share their location and moving information. When a node encounters another node, each node exchanges its location and historical moving information and decides whether it delivers its packets to the encountering node by calculating the delivery predictability, which is based on the historical encounter duration time and buffer situation. Based on this prediction, the node will make a wise decision to deliver the packets or not in both the spray phase and wait phase. In the wait phase of the original Spray and Wait, a node with the last copy has to wait until it encounters the destination, the node will not hand over the last copy to any nodes that might have more chances to encounter the destination, so it might waste some opportunity and keep the buffer out of space. In this scheme, we make an effort to let nodes exchange SV (Summary Vector) once a node with the last one copy encounters a node. We calculate the total utility according to the buffer utility and delivery probability. If the total utility is higher than a given threshold, the node will hand over the last copy to it.

\subsection{Delivery Predictability Calculation}

In the spray phase of original Spray and Wait routing, for each message originating at a source node, $L$ message copies are initially sprayed and relayed by nodes. An optimal Spray and Wait scheme-Binary Spray and Wait (BSW) is proposed in [8] to speed up the spray phase and improve the routing performance, where any node with $n \geq 1$ copies hands over half copies to the encountered node until $n=1$. However, if the relay is a very inactive node, which does not contact with other nodes, handing over half the copies to such a node means half of the relay chances will be wasted. In order to overcome this problem, we introduce a novel scheme to the spray phase, where we set up a delivery probability to the destinations for each node as the ProPHET routing [11] does, and nodes exchange different numbers of 
copies to each other according to their delivery probabilities $P_{(a, b)} \in[0,1]$, that is, when $P_{(a, b)}$

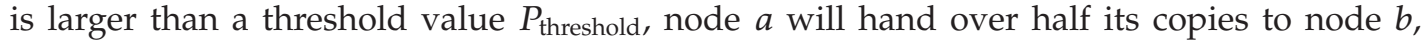
otherwise, it will only hand over one copy to node $b$. The delivery probabilities are updated as follows.

(1) Whenever a node is encountered, the delivery predictability is updated as (2.1), where $P_{\text {init }}$ is an initialization constant. It is recommended in [18] that the referential value of $P_{\text {init }}$ is 0.75 :

$$
P(a, b)=P(a, b)_{\text {old }}+\left(1-P(a, b)_{\text {old }}\right) \times P_{\text {init }}
$$

(2) The aging equal is shown in (2.2), where $\gamma \in(0,1]$ is the aging constant and $k$ is the number of time units that has elapsed since the last time the metric was aged:

$$
P(a, b)=P(a, b)_{\text {old }} \times \gamma^{k}
$$

(3) Transitive affection is shown in (2.3), where $\beta$ is a scaling constant that decides how large impact the transitivity should have on the delivery predictability. It is recommended in [18] that the referential value of $\beta$ is 0.25 :

$$
P(a, c)=P(a, c)_{\mathrm{old}}+\left(1-P(a, c)_{\mathrm{old}}\right) \times P(a, b) \times P(b, c) \times \beta .
$$

In real opportunistic networks, the communication range of nodes, moving speed, and bandwidth may be different. When the network is in an unstable situation, such that the nodes' moving speeds are too fast and have different communication ranges, the links will interrupt frequently. In this case, the nodes encounter each other from time to time according to the link's situation. The number of nodes encountering cannot reflect the real ability of communication between nodes anymore. Based on this observation, we revise (2.1) and (2.2) using the continuous time $t$ to calculate the delivery as that of [19], where $\tau$ is a constant, and $t$ is the time that has elapsed since the last encounter time:

$$
P_{t}=P_{\text {old }} \times e^{-\tau t}
$$

Compared with (2.2) and (2.4), let

$$
t=k u, \quad P_{t}(a, b)=P(a, b),
$$

then

$$
\begin{aligned}
\left(e^{-\tau}\right)^{t}=\left(\gamma^{1 / u}\right)^{t} & =\left(e^{(1 / u) \ln \gamma}\right)^{t} \mid P_{\text {old }} \neq 0, \\
\tau & =-\frac{1}{u} \ln \gamma,
\end{aligned}
$$


and then (2.1) can be rewritten as follows:

$$
\begin{aligned}
P(a, b) & =1-e^{-c t_{\text {up }}}\left(1-P(a, b)_{\mathrm{old}}\right), \\
c & =-\frac{1}{u^{\prime}} \ln \left(1-P_{\text {init }}\right) .
\end{aligned}
$$

In this way, the discrete-time signal is changed into the continuous-time signal. Therefore, the noise, which is introduced by different encounter communication range and moving speeds, can be removed by certain filter.

\subsection{Optimal Node Selection with Buffer Management}

In this subsection, we try to maximize the average delivery rate by using a buffer management. In opportunistic networks, nodes have finite buffer space, so they must eventually discard old copies to make room for new requests. Normally, copies will be discarded when the Time to Live (TTL) is elapsed. If the TTL elapsed before the nodes encounter any nodes, the copies will be dropped; otherwise, a decision of which copies should be dropped must be made when the buffer is filled up. Thus, the encountering interval of nodes should be considered. The encountering interval between nodes depends on the value of the mobility model. We assume that there is enough time to exchange their packets. The encountering time $(T)$ between nodes is defined as the time it takes them to first come within transmission rang $\left(R=\min \left(r_{a}, r_{b}\right)\right)$. Based on the experimental study, it has been shown that the meeting time of some random-based mobility models like Random Walk, Random Waypoint, and Random Direction is exponentially distributed or has at least an exponential tail, with parameter $\lambda=1 / E(T)$, where $E(T)$ denotes the expectation of a random variable $T$. We use these mobility models for our test scenarios. And, then, the probability that a copy of a message $j$ will not be delivered is equal to the probability that the next encountering time with the destination node is greater than the remaining Time to Live $R_{j}$ (TTL) for message $j$. That is $\exp \left(-\lambda R_{j}(\mathrm{TTL})\right)$. Based on this model, it has been proved that in order to maximize the average delivery rate, the optimal policy of buffer management should drop the message with the lowest probability to delivery [14]. The optimal policy of buffer management uses the Epidemic Routing, whose number of message copies is uncontrolled. Different from that, we employ a fixed number $L$ of copies for messages. The probability that the message will not be delivered can be derived as follows:

$$
P_{j}(\text { undelivered })=\exp \left(-\lambda n_{j} R_{j}(\mathrm{TTL})\right)
$$

Here, $n_{j}$ is the total number of copies of message $j$ in network. And the probability of a message being delivered is:

$$
P_{j}(\text { delivered })=\frac{m_{j}}{N-1}
$$


where $N$ is the number of nodes in the network and $m_{j}$ is the number of nodes that have ever stored message $j$. Then, the probability of message that will be delivered can be derived as follows:

$$
\begin{gathered}
P_{j}=P_{j}(\text { delivered })\left(1-\exp \left(-\lambda n_{j} R_{j}(\mathrm{TTL})\right)\right)+P_{j}(\text { delivered }), \\
P_{j}=\left(1-\frac{m_{j}}{N-1}\right)\left(1-\exp \left(-\lambda n_{j} R_{j}(\mathrm{TTL})\right)\right)+\frac{m_{j}}{N-1} .
\end{gathered}
$$

Hence, the maximum of average delivery rate is achieved by maximizing $P_{j}$, then we can maximize the average delivery rate. Taking the derivation of (2.12) with respect to $n_{j}$ :

$$
\frac{\partial P_{j}}{\partial n_{j}}=\left(1-\frac{m_{j}}{N-1}\right) \lambda R_{j} \exp \left(-\lambda n_{j} R_{j}(\mathrm{TTL})\right) \Delta n_{j}
$$

From (2.13), the best drop decision is to drop the message $j$ satisfying:

$$
j_{\min }=\underset{j}{\arg \min }\left[\left(1-\frac{m_{j}}{N-1}\right) \lambda R_{j} \exp \left(-\lambda n_{j} R_{j}(\mathrm{TTL})\right)\right]
$$

Since we are using the fixed number $L$ of the copies, when the proposed routing comes to waiting phase, it means that the nodes have only one copy of the message $j$. For the worst case, none of them contacts destination node. Replacing $n_{j}$ with the total number of copies $L$ for (2.14), we get

$$
j_{\min }=\underset{j}{\arg \min }\left[\left(1-\frac{m_{j}}{N-1}\right) \lambda R_{j} \exp \left(-\lambda L R_{j}(\mathrm{TTL})\right)\right]
$$

We define the buffer utility as follows:

$$
U_{j}=\left(1-\frac{m_{j}}{N-1}\right) \lambda R_{j} \exp \left(-\lambda L R_{j}(\mathrm{TTL})\right)
$$

where value of $m_{j}$ is a global state of the message in the network. We can calculate it by using the local information. Suppose that

$$
m_{j}=\bar{m}_{j}=E(M(T)),
$$

where $M(T)$ is a random variable, which follows the approximated of a Gaussian distribution.

In the fixed number copies' routing, the success delivery rate depends on the threshold of the number of copies and the spray strategy. Multiple-copy routing utilizes multiple paths to transfer packets. Therefore, the node with larger delivery predictability should have more copies of the packet. While the source spray and binary spray strategies used in Spray and Wait routing do not consider the different utilities of the nodes. They spray the packets 


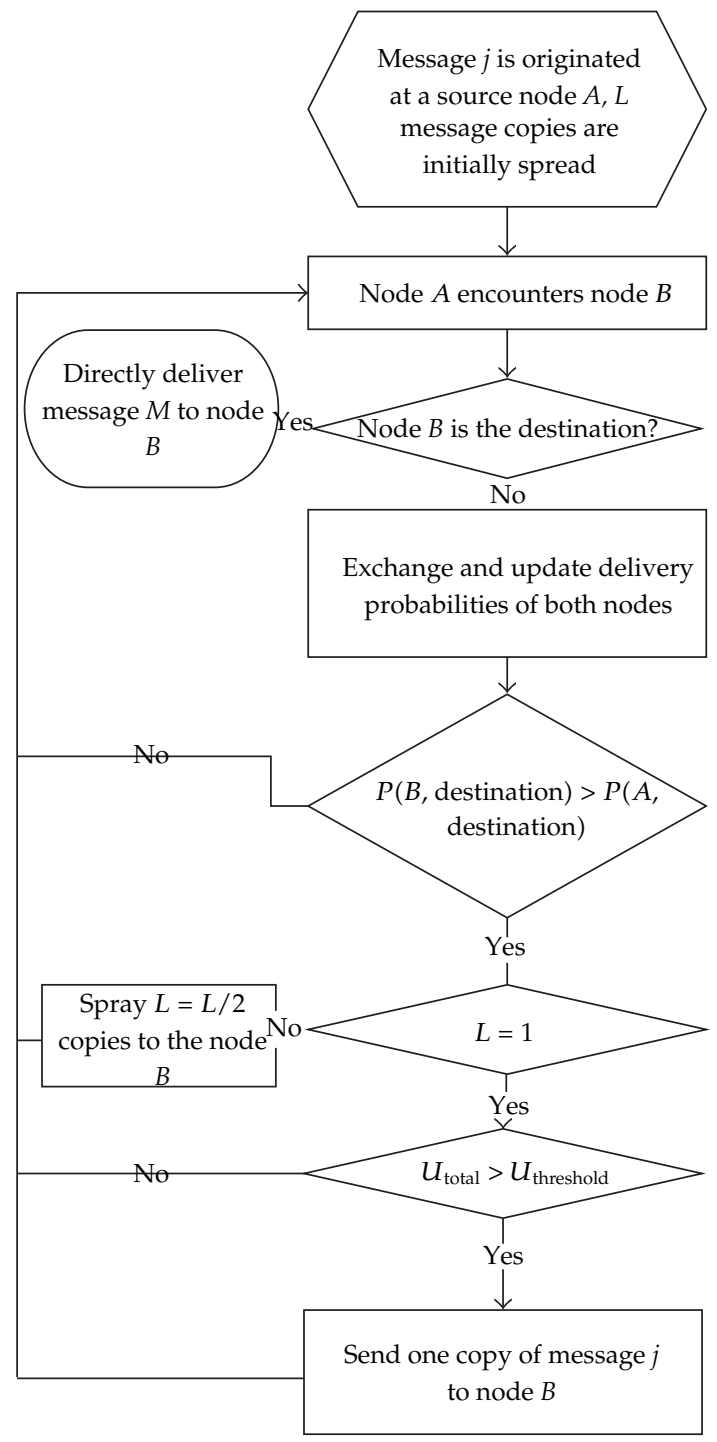

Figure 1: Flow chart of the proposed routing.

equally for each node. According to the analysis in Section 2.3, we apply a simple spray strategy based on mean delivery predictability to the routing. Set $B$ as the sum of average contact and intercontact time between encounters of node pairs. We calculate the mean delivery predictability $\bar{P}$ as follows:

$$
\bar{P}_{(a, b)}=\frac{1}{B} \int_{0}^{B} P_{\text {init }(a, b)} \times e^{-\lambda t} d t
$$

when $P>\bar{P}$, half copies $(L / 2)$ will be transferred to the encountering node. When $P<\bar{P}$, only one copy will be transferred to the encountering node. 
Table 1: Simulation environment parameters.

\begin{tabular}{lc}
\hline Simulation parameters & Simulation values \\
\hline Map size & $4500 \mathrm{~m} \times 3400 \mathrm{~m}$ \\
Packet transmission speed & $250 \mathrm{kBps}(2 \mathrm{Mbps})$ \\
Number of nodes & \\
$\quad$ Pedestrian & 60 \\
$\quad$ Bicycles & 30 \\
$\quad$ Vehicles & 30 \\
Node movement & Shortest Path Map Based Movement \\
Speed & \\
$\quad$ Pedestrian & $0.5-1.5 \mathrm{~m} / \mathrm{s}$ \\
$\quad$ Bicycles & $1.4-4 \mathrm{~m} / \mathrm{s}$ \\
Vehicles & $2.7-13.9 \mathrm{~m} / \mathrm{s}$ \\
Transmission range & $10 \mathrm{~m}$ \\
Packet size & $500 \mathrm{kB}-1 \mathrm{MB}$ \\
Message generation interval & $25 \mathrm{~s}, 35 \mathrm{~s}$ \\
\hline
\end{tabular}

In the wait phase, the original Spray and Wait store message in the buffer until the destination is reached. Sometimes, however, the encountering node may have more chance to encounter the destination, we consider delivering the last copy to the encountering node with a larger delivery probability. Since this policy may lead to no convergence (none of the nodes reach the destination before TTL), we consider the following process.

By combining (2.7) and (2.16), we construct a total utility of buffer utility and delivery probability as follows:

$U_{\text {total }}=\delta U_{j}+\psi P(a, b)=\delta\left[1-e^{-c t_{\text {up }}}\left(1-P(a, b)_{\text {old }}\right)\right]+\psi\left(1-\frac{m_{j}}{N-1}\right) \lambda R_{j} \exp \left(-\lambda L R_{j}(\mathrm{TTL})\right)$

where $\delta$ and $\psi$ are the weighted factors that represent the impact of buffer utility and delivery probability on the total utility, respectively.

If the total utility $U_{\text {total }}$ is larger than a given threshold $U_{\text {threshold, }}$ the last copy will be sent to the encountering node, otherwise it will be kept in the buffer until the TTL expires. The flow of the scheme is shown in Figure 1.

\section{Simulation and Analysis}

This section evaluates the performance of the proposed routing by modifying and developing the traditional Spray and Wait routing in the ONE $[15,20]$ simulator. We consider a scenario with three classes of nodes, pedestrians, bicycles, and vehicles. The details of the simulation parameters are listed in Table 1.

In the performance evaluation, we compare the proposed protocol with three representative routing protocols: Epidemic Routing (ER), original Spray and Wait (SNW), and ProPHET routing (PRO), respectively. We run all these routings in the same scenario with the above parameters and compare their performance with regard to the success delivery 


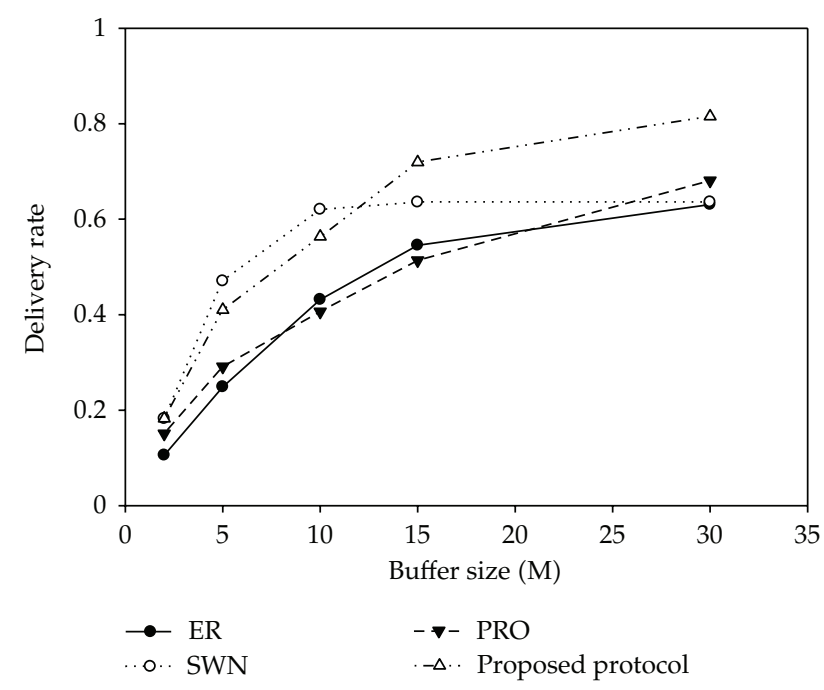

Figure 2: Delivery rate with different buffer size.

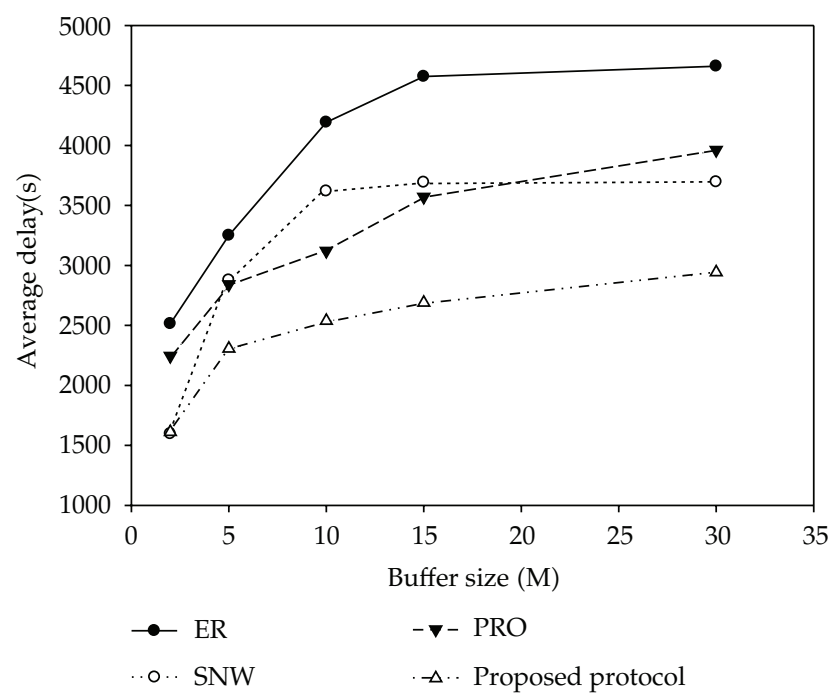

Figure 3: Average delay with different buffer size.

rate and delivery delay under different buffer size, TTL, and total number of messages, respectively.

Figures 2 and 3 show the delivery rate and average delay with variant buffer size for ER, SNW, and PRO in comparison with the proposed optimal probability choice routing protocol with buffer management. Among these routing protocols, the bigger buffer size show the better performance of all protocols, and the performances of SNW and the proposed protocol are better than PRO and ER. This result is due to the use of the limited number of copies among these routings. Note that the proposed protocol provides a higher delivery rate than SNW when the buffer size is larger than $12 \mathrm{M}$. This result validates the effectiveness of the proposed buffer management policy in Section 2.4 . 


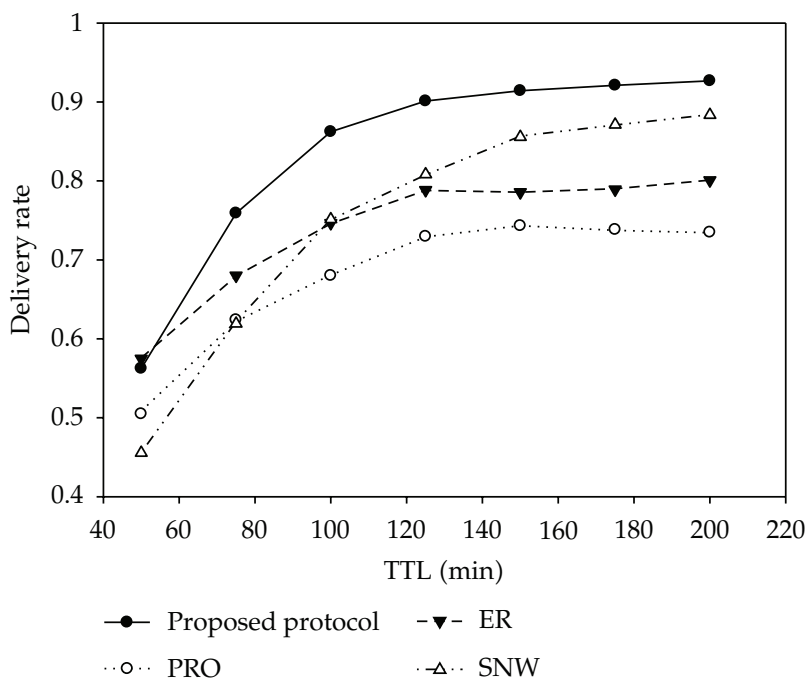

Figure 4: Delivery rate with different TTL.

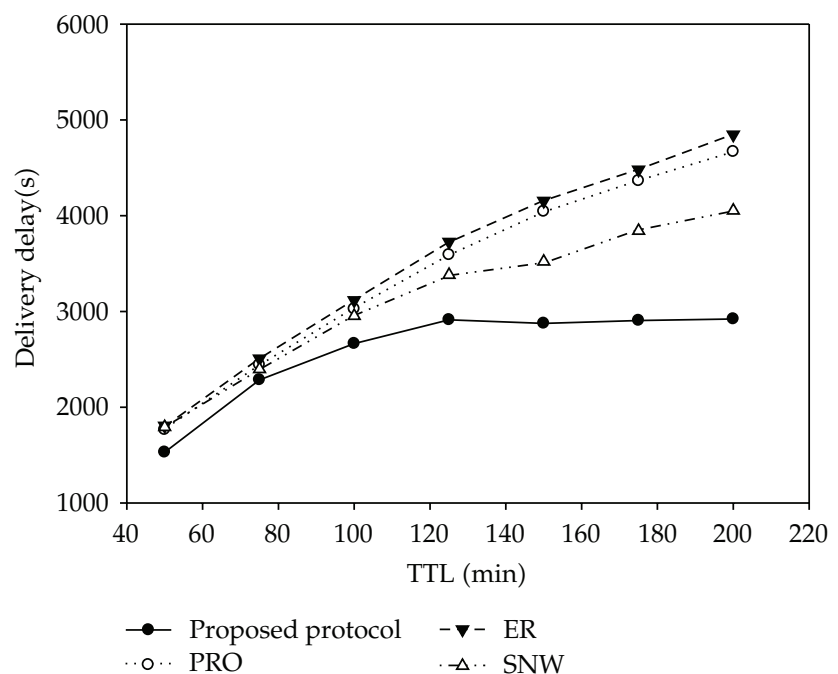

Figure 5: Delay with different TTL.

Figures 4 and 5 show the performance under different TTL. The figures show that the proposed protocol outperforms the other three routing algorithms. Along with the increase of TTL, the delivery rates and the average delivery delays of all the four routings rise gradually. The proposed protocol achieves the largest delivery rate and the shortest delay for all TLL scenarios. This is reasonable because large TTL brings more time for the copies to stay in the relay nodes without discarding, and this helps to increase the success delivery rate, while the long-time staying in nodes will lead to lack of buffer spaces and large average delivery delay, when buffer spaces run out, copies will be discarded again, which will lead to a reduction in success delivery rate. That is, tradeoff is offered in terms of the TTL and buffer space. 


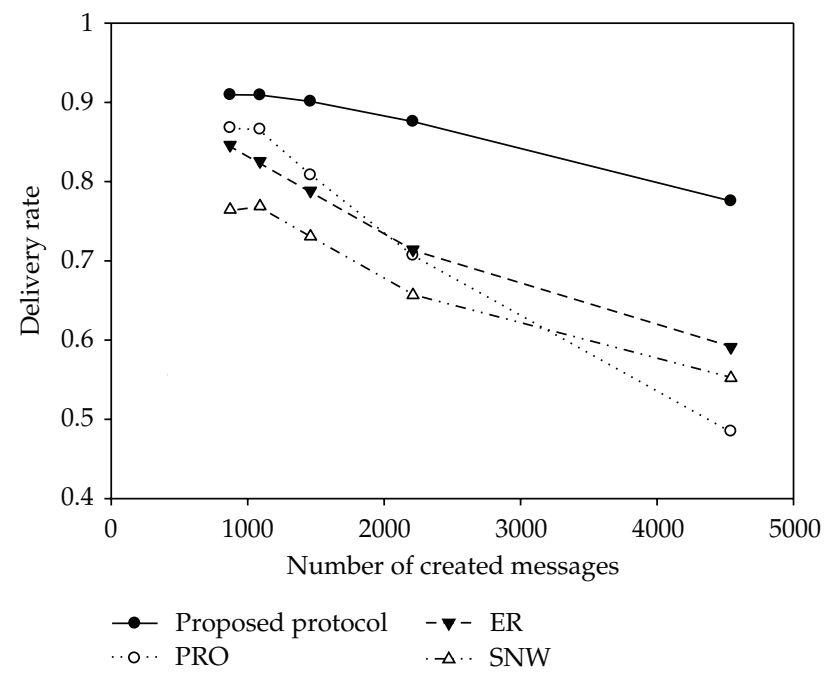

Figure 6: Delivery rate with number of messages.

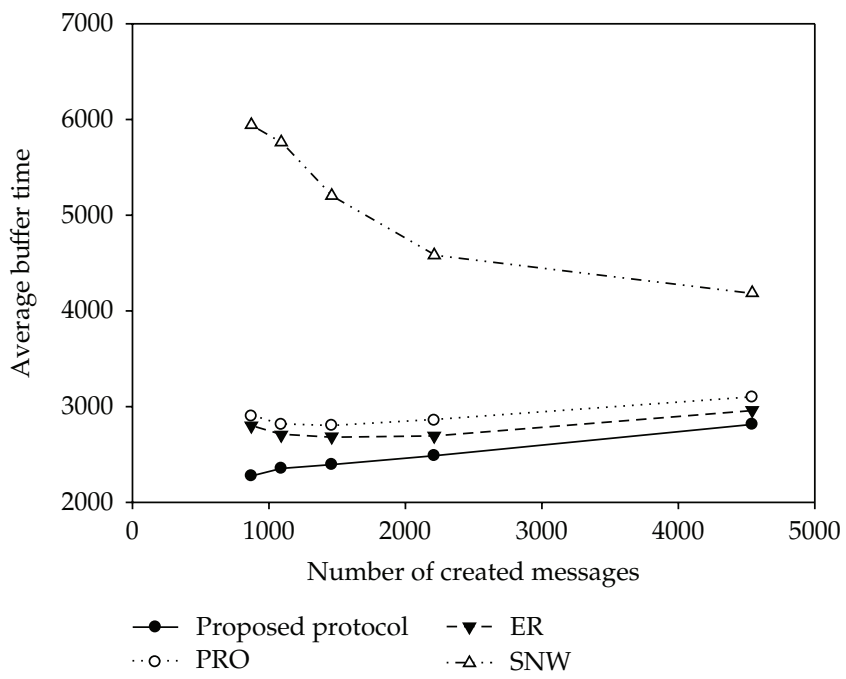

Figure 7: Average buffer time with number of messages.

Although both the proposed protocol and the SNW require more copies than the other schemes, our protocol outperforms the latter as the buffer size is increased. This is because we use directly delivering in the wait phase to transmit the last one copy to the node with a higher delivery probability to destination.

Figures 6, 7, 8, and 9 describe the average delivery rate, average buffer time, the delivery delay, and overhead versus the total number of message. As the traffic increases, the delivery rates and overhead of all the routing protocols decrease, while the average buffer time and delivery delay increase eventually. Overall, the delivery rate of the proposed protocol is the highest one and it is more robust than the other routing protocols, and the average buffer time and overhead of the proposed protocol is kept in a very low level. The 


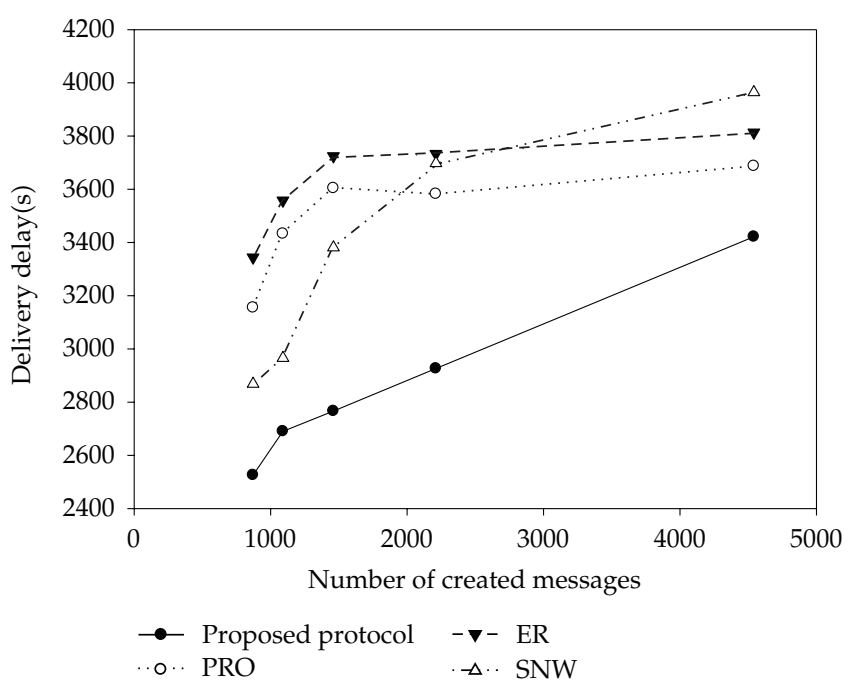

Figure 8: Delivery delay with number of messages.

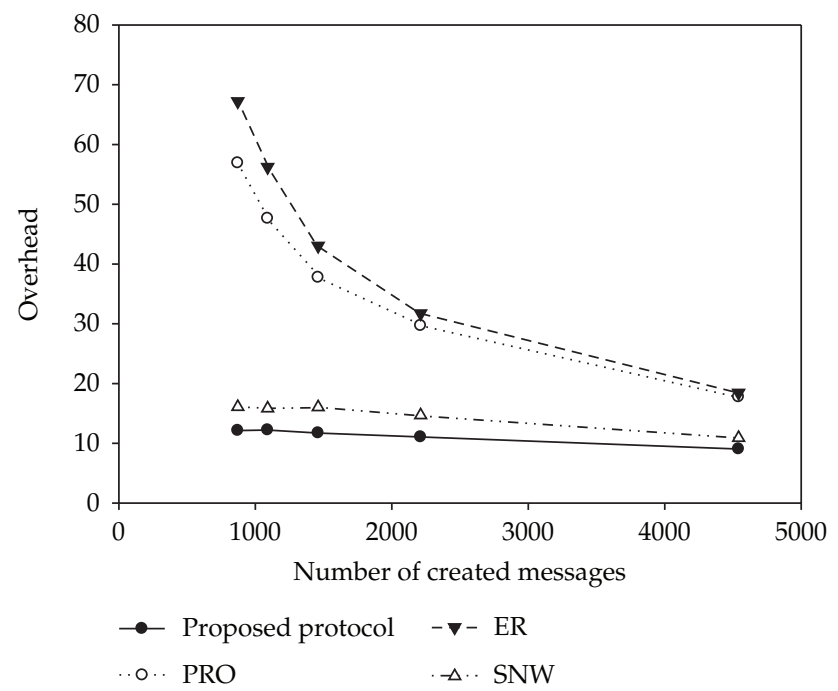

Figure 9: Overhead with number of messages.

reason is as follows. Firstly, we use continuous encounter time to describe the encounter opportunity, which makes it more precisely to describe the encounter opportunity. Secondly, we provide different numbers of exchanging copies to the nodes according to their delivery probabilities to the destination in the spray phase, the node transfers more copies to the node with higher delivery predictability. It takes full advantage of the knowledge about the historical encountering, and the delivery predictability reflects the node's real mobility and transfer ability more precisely and thus yields a faster transfer for the packet to the destination node. Finally, taking the buffer management and the delivery probability into account in final waiting phase will gain more buffer space the buffer and thus reduce the average buffer time and increase the opportunity of finding the destination. 


\section{Conclusion}

Opportunistic networks aim to provide reliable communications in an intermittently connected environment. The major challenge here is to route messages without an end-toend connection. To deal with the unpredictability in connections and network partitions, we propose the probability choice routing protocol with buffer management for opportunistic networks in this paper. In the proposed protocol, a delivery probability based on continuous encountering duration time is set up such that each node can choose a better node as its relay in spray phase, and a total utility of buffer management utility and delivery probability is taken into consideration for delivering the last copy to the encountering node. Extensive results are provided to evaluate the proposed routing protocol with ONE simulator. Simulation experiments indicate that the proposed routing protocol outperforms the existing routing solutions thanks to its ability to maximize the delivery rate and minimize the delivery delay. Future research topic includes the extension to the real-life mobility.

\section{Acknowledgments}

This work is supported by National Natural Science Foundation of China under Grants no. 61172087, no. 61002012, and the Natural Science Foundation of Guangdong Province under Grant no. 06300923.

\section{References}

[1] L. Pelusi, A. Passarella, and M. Conti, "Opportunistic networking: data forwarding in disconnected mobile ad hoc networks," IEEE Communications Magazine, vol. 44, no. 11, pp. 134-141, 2006.

[2] K. Fall, "A delay-tolerant network architecture for challenged internets," in Proceedings of the Conference on Applications, Technologies, Architectures, and Protocols for Computer Communication (SIGCOMM '03), pp. 27-34, August 2003.

[3] I. F. Akyildiz, B. Akan, C. Chen, J. Fang, and W. Su, "InterPlaNetary Internet: state-of-the-art and research challenges," Computer Networks, vol. 43, no. 2, pp. 75-112, 2003.

[4] T. Spyropoulos, K. Psounis, and C. S. Raghavendra, "Multiple-copy routing in intermittently connected mobile networks," Tech. Rep. CENG-2004-12, University of Southern California, Los Angeles, Calif, USA, 2004.

[5] W. Huang and S. Chen, "Epidemic metapopulation model with traffic routing in scale-free networks," Journal of Statistical Mechanics, vol. 2011, Article ID P12004, 12 pages, 2011.

[6] S. Chen, W. Huang, C. Cattani, and G. Altieri, "Traffic dynamics on complex networks: a survey," Mathematical Problems in Engineering, vol. 2012, Article ID 732698, 23 pages, 2012.

[7] A. Vahdat and D. Becker, "Epidemic routing for partially-connected ad hoc networks," Tech. Rep. CS-2000-06, Duke University Durham, Durham, NC, USA, 2000.

[8] T. Spyropoulos, K. Psounis, and C. S. Raghavendra, "Single-copy routing in intermittently connected mobile networks," in Proceedings of the 1st Annual IEEE Communications Society Conference on Sensor and Ad Hoc Communications and Networks (SECON '04), pp. 235-244, October 2004.

[9] T. Spyropoulos, K. Psounis, and C. S. Raghavendra, "Spray and wait: efficient routing in intermittently connected mobile networks," in Proceedings of ACMSIGCOMM workshop on Delay Tolerant Networking (WDTN'05), 2005.

[10] M. Li, "Approximating ideal filters by systems of fractional order," Computational and Mathematical Methods in Medicine, vol. 2012, Article ID 365054, 6 pages, 2012.

[11] H. A. Nguyen, S. GiordanoS, and A. Puiatti, "Probabilistic routing protocol for intermittently connected mobile ad hoc network," in Proceedings of IEEE International Symposium on a World of Wireless, Mobile and Multimedia Networks, Espoo, Finland, 2007. 
[12] J. Burgess, B. Gallagher, D. Jensen, and B. N. Levine, “MaxProp: routing for vehicle-based disruptiontolerant networks," in Proceedings of the 25th IEEE International Conference on Computer Communications (INFOCOM '06), Barcelona, Spain, April 2006.

[13] T. Anindya, D. Rajkumar, K. Humayun, and C. S. Gholamali, "TTL based routing in opportunistic networks," Journal of Network and Computer Applications, vol. 34, no. 5, pp. 1660-1670, 2011.

[14] A. Krifa and C. Barakat, "An optimal joint scheduling and drop policy for delay tolerant networks," in Proceeding of the International Symposium on World of Wireless, Mobile and Multimedia Networks (WoWMoM '08), Newport Beach, Calif, USA, 2008.

[15] S. C. Nelson, M. Bakht, and R. Kravets, "Encounter-based routing in DTNs," in Proceedings of the 28th Conference on Computer Communications (INFOCOM '09), pp. 846-854, Rio de Janeiro, Brazil, April 2009.

[16] R. Santos, J. Orozco, and F. Sergio, "A real-time analysis approach in opportunistic networks," ACM SIGBED Review, vol. 8, no. 3, pp. 40-43, 2011.

[17] M. Li, C. Cattani, and S. Y. Chen, "Viewing sea level by a one-dimensional random function with long memory," Mathematical Problems in Engineering, vol. 2011, Article ID 654284, 13 pages, 2011.

[18] M. Li and W. Zhao, "Visiting power laws in cyber-physical networking systems," Mathematical Problems in Engineering, vol. 2012, Article ID 302786, 13 pages, 2012.

[19] M. Li and W. Zhao, "Representation of a stochastic traffic bound," IEEE Transactions on Parallel and Distributed Systems, vol. 21, no. 9, Article ID 5342414, pp. 1368-1372, 2010.

[20] J. Karvo and J. Ott, "Time scales and delay-tolerant routing protocols," in Proceedings of the 3rd ACM workshop on Challenged networks (CHANTS '08), San Francisco, Calif, USA, 2008. 


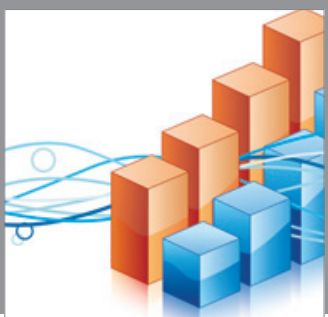

Advances in

Operations Research

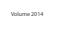

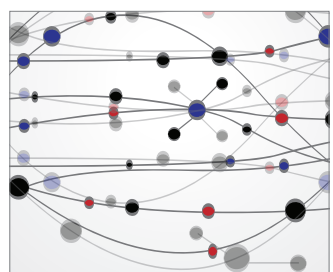

\section{The Scientific} World Journal
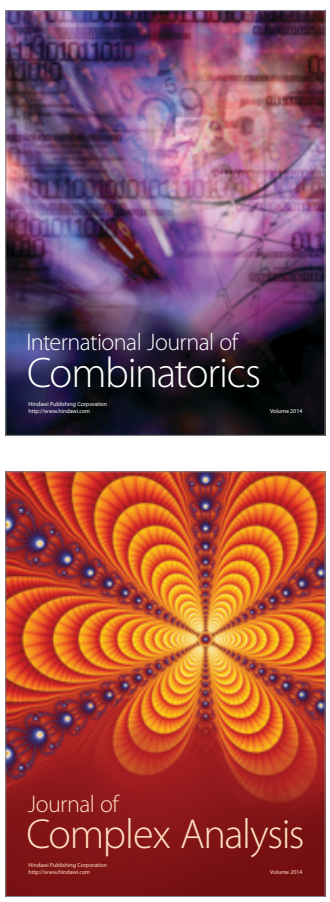

International Journal of

Mathematics and

Mathematical

Sciences
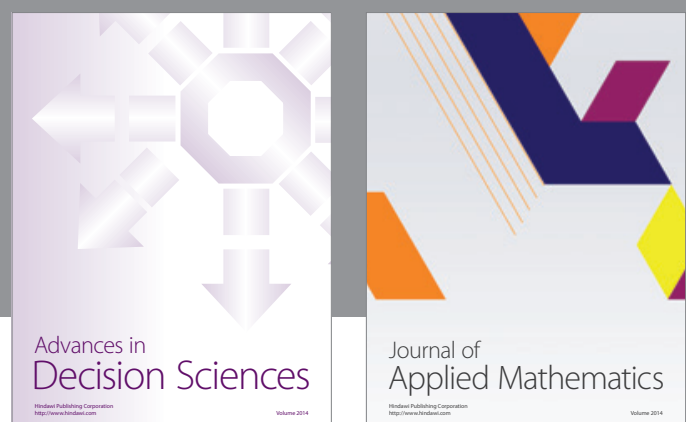

Journal of

Applied Mathematics
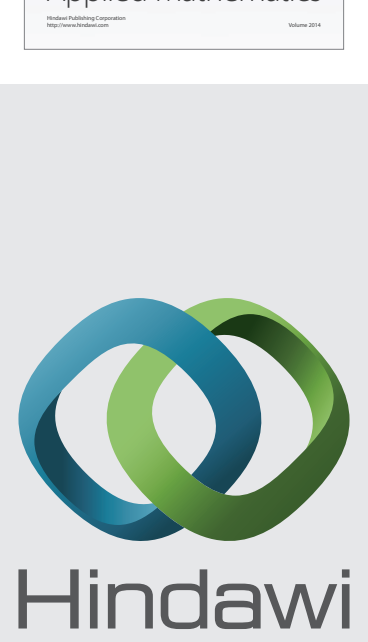

Submit your manuscripts at http://www.hindawi.com
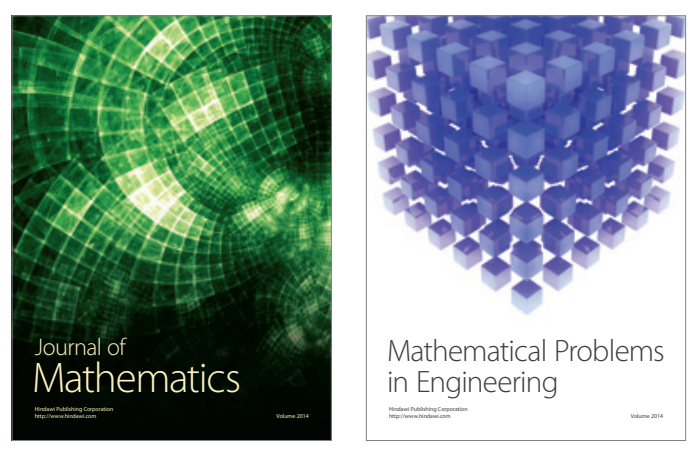

Mathematical Problems in Engineering
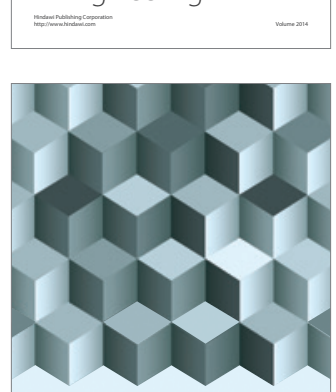

Journal of

Function Spaces
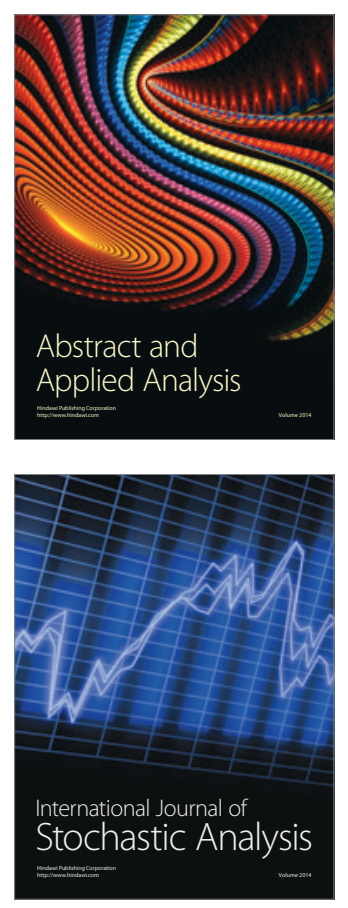

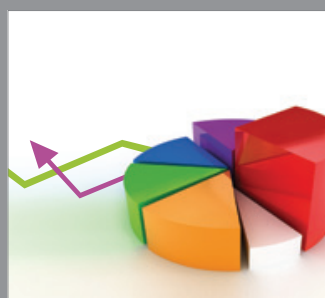

ournal of

Probability and Statistics

Promensencen
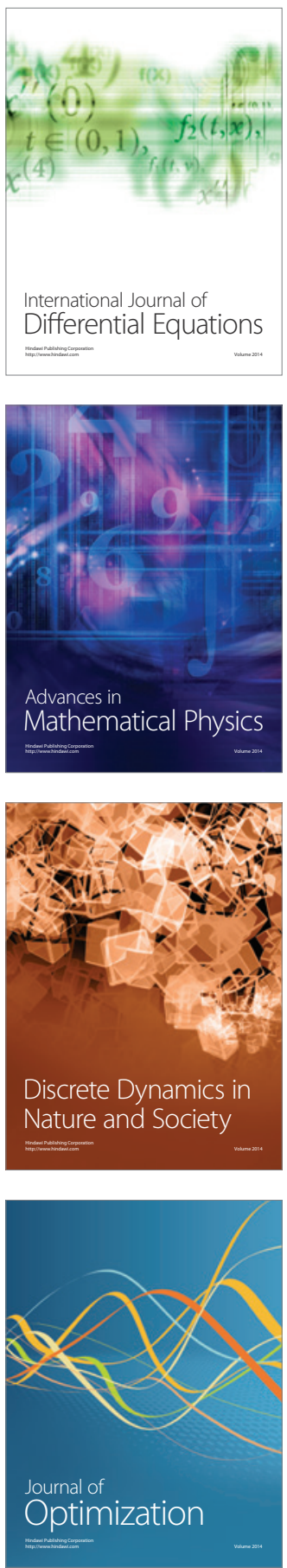